\title{
Fourier expansion and integral representation generalized Apostol-type Frobenius-Euler polynomials
}

\author{
Alejandro Urieles ${ }^{1 *}$ (D), William Ramírez ${ }^{2}$, María José Ortega² and Daniel Bedoya ${ }^{3}$
}

${ }^{*}$ Correspondence:

alejandrourieles@mail.uniatlantico.edu.c

${ }^{1}$ Programa de Matemáticas,

Universidad del Atlántico, Km 7 Vía

Pto, Barranquilla, Colombia

Full list of author information is

available at the end of the article

\begin{abstract}
The main purpose of this paper is to investigate the Fourier series representation of the generalized Apostol-type Frobenius-Euler polynomials, and using the above-mentioned series we find its integral representation. At the same time applying the Fourier series representation of the Apostol Frobenius-Genocchi and Apostol Genocchi polynomials, we obtain its integral representation. Furthermore, using the Hurwitz-Lerch zeta function we introduce the formula in rational arguments of the generalized Apostol-type Frobenius-Euler polynomials in terms of the Hurwitz zeta function. Finally, we show the representation of rational arguments of the Apostol Frobenius Euler polynomials and the Apostol Frobenius-Genocchi polynomials.
\end{abstract}

Keywords: Generalized Apostol Frobenius-Euler polynomials; Hurwitz zeta function; Fourier expansion; Generalized Apostol Frobennius-Euler numbers

\section{Introduction}

The Fourier series of a periodic function can be written exponentially as (see [9, p. 19, Eq. (2.2)])

$$
f(x)=\sum_{n=-\infty}^{\infty} a_{n} e^{i n w x} ; \quad\left(w=\frac{2 \pi}{T}\right)
$$

the coefficients $a_{n}$ and $\bar{a}_{n}$ are computed by

$$
a_{n}=\frac{1}{T} \int_{0}^{\frac{2 \pi}{w}} e^{-i n w t} f(t) d t \quad \text { and } \quad \bar{a}_{n}=\frac{1}{T} \int_{0}^{\frac{2 \pi}{w}} e^{i n w t} f(t) d t
$$

Here $\bar{a}_{n}$ is the complex conjugate of $a_{n}$.

The Frobenius-Euler polynomials and the Frobenius-Euler numbers play an important role in the number of theories and classical analysis. In particular, the Frobenius-Euler polynomials appear in the integral representation of differentiable periodic functions since they are employed for approximating such functions in terms of polynomials (see [1, 4$6,10,13,19])$. The Frobenius-Euler polynomials $H_{n}(x ; u)$ in the variable $x$ are defined by

(c) The Author(s) 2020. This article is licensed under a Creative Commons Attribution 4.0 International License, which permits use, sharing, adaptation, distribution and reproduction in any medium or format, as long as you give appropriate credit to the original author(s) and the source, provide a link to the Creative Commons licence, and indicate if changes were made. The images or other third party material in this article are included in the article's Creative Commons licence, unless indicated otherwise in a credit line to the material. If material is not included in the article's Creative Commons licence and your intended use is not permitted by statutory regulation or exceeds the permitted use, you will need to obtain permission directly from the copyright holder. To view a copy of this licence, visit http://creativecommons.org/licenses/by/4.0/. 
means of the generating function (see [11, p. 268])

$$
\left(\frac{1-u}{e^{z}-u}\right) e^{x z}=\sum_{n=0}^{\infty} H_{n}(x ; u) \frac{z^{n}}{n !}, \quad|z|<|\log (u)|,
$$

when $x=0, H_{n}(u)$ denotes the so-called Frobenius-Euler numbers. $H_{n}(x ;-1)=E_{n}(x)$ denotes the Euler polynomials (see $[16,17]$ ).

The Fourier series representation of the Frobenius-Euler polynomials are given by (see [2, p. 8, Corollary 4])

$$
H_{n}(x ; u)=\frac{(u-1)}{u} u^{x} n ! \sum_{k \in \mathbb{Z}} \frac{e^{2 \pi i k x}}{[2 \pi i k+\log (u)]^{n+1}},
$$

if $u, \in \mathbb{C}$ with $u \neq 1$, and $0<x<1$.

The Frobenius-Genocchi polynomials $\mathfrak{G}_{n}^{F}(x ; u)$ in the variable $x$ are defined by the generating function (see [2, p. 3, Definition 3])

$$
\left(\frac{1-u}{e^{z}-u}\right) z e^{x z}=\sum_{n=0}^{\infty} \mathfrak{G}_{n}^{F}(x ; u) \frac{z^{n}}{n !} ; \quad|z|<|\log (u)|
$$

when $x=0, \mathfrak{G}_{n}^{F}(u)$ denotes the so-called Frobenius-Genocchi numbers, then the Fourier series representation of (3) is given by

$$
\mathfrak{G}_{n}^{F}(x ; u)=\frac{(u-1)}{u} u^{x} n ! \sum_{k \in \mathbb{Z}} \frac{e^{2 \pi i k x}}{[2 \pi i k+\log (u)]^{n}} .
$$

Some authors have proved a Fourier series and integral representations for the ApostolEuler polynomials and Apostol-Bernoulli polynomials by using the Lipschitz summation formula (see [14]). On the other hand, in [3] using the Cauchy residue theorem in the complex plane, the author proved a Fourier series for the Apostol-Bernoulli, ApostolGenocchi and Apostol-Euler polynomials. Other authors revealed a Fourier expansion for Apostol Frobenius-Euler polynomials and Apostol Frobenius-Genocchi polynomials (cf. [2]). We recently studied the Fourier expansions for higher-order Apostol-Genocchi, Apostol-Bernoulli and Apostol-Euler polynomials (see [8]).

In this paper, we obtained the Fourier expansion of generalized Apostol-type FrobeniusEuler polynomials and its integral representation to show the explicit formula at rational arguments for these polynomials in terms of the Hurwitz zeta function. Also, we will show the integral representation of Apostol Frobenius-Euler, Apostol Frobenius-Genocchi, Frobenius-Genocchi, Frobenius-Euler polynomials, and give a new representation for the polynomials of Apostol-Euler and Apostol-Genocchi and some formulas in rational arguments.

This article is organized as follows. Section 2 contains the basic background about polynomials of Apostol-type Frobenius-Euler, Apostol Frobenius-Genocchi, FrobeniusGenocchi, Frobenius-Euler and generalized Apostol-type Frobenius-Euler polynomials in the variable $x$, parameters $\lambda, u \in \mathbb{C}, a, b, c \in \mathbb{R}^{+}$. In Sect. 3 are revealed the Fourier expansions for the generalized Apostol-type Frobenius-Euler polynomials, and several corollaries for other families of known polynomials. In Sect. 4, we obtain the integral representation of generalized Apostol-type Frobenius-Euler polynomials, that is, Theorem 4.1. 
At the same time, we achieved the integral representation of the Frobenius-Euler and Frobenius-Genocchi polynomials, that is, Theorems 4.2 and 4.3. Also, Sect. 5 secures the explicit formula at rational arguments in terms of Hurwitz zeta function of generalized Apostol-type Frobenius-Euler polynomials, that is, Theorem 5.1. Finally, we obtained the formula in rational arguments for the Frobenius-Euler and Frobenius-Genocchi polynomials, that is, Theorems 5.2 and 5.3 , respectively.

\section{Background and previous results}

Throughout this paper, we use the following standard notions: $\mathbb{N}=\{1,2, \ldots\}, \mathbb{N}_{0}=$ $\{0,1,2, \ldots\}, \mathbb{Z}$ denotes the set of integers, $\mathbb{R}$ denotes the set of real numbers, $\mathbb{C}$ denotes the set of complex numbers. For the complex logarithm, we consider the principal branch.

On the other hand, we have the well-known integral formula (see [15, p. 2198, Eq. (3.2)])

$$
\int_{0}^{\infty} t^{n} e^{-a t} d t=\frac{n !}{a^{n+1}}, \quad n \in \mathbb{N}_{0} ; \Re e(a)>0
$$

It is well known that Apostol-type Frobenius-Euler polynomials $H_{n}(x ; u ; \lambda)$ in the variable $x$ are defined by means of the generating function (see [4, p. 164, Eq. (1.1)])

$$
\left(\frac{1-u}{\lambda e^{z}-u}\right) e^{x z}=\sum_{n=0}^{\infty} H_{n}(x ; u ; \lambda) \frac{z^{n}}{n !}, \quad|z|<\left|\log \left(\frac{\lambda}{u}\right)\right|
$$

The Fourier series representation of the Apostol-type Frobenius-Euler polynomials is given by (see [2, p. 5 , Theorem 1])

$$
H_{n}(x ; u ; \lambda)=\frac{(u-1)}{u} \frac{u^{x}}{\lambda^{x}} n ! \sum_{k \in \mathbb{Z}} \frac{e^{2 \pi i k x}}{\left[2 \pi i k-\log \left(\frac{\lambda}{u}\right)\right]^{n+1}} .
$$

Also, the Fourier series representation of Apostol-type Frobenius-Genocchi polynomials is given by (see [2, p. 13, Theorem 11])

$$
\mathfrak{G}_{n}^{F}(x ; u ; \lambda)=\frac{(u-1)}{u} \frac{u^{x}}{\lambda^{x}} n ! \sum_{k \in \mathbb{Z}} \frac{e^{2 \pi i k x}}{\left[2 \pi i k-\log \left(\frac{\lambda}{u}\right)\right]^{n}},
$$

which makes sense if $u, \lambda \in \mathbb{C}$ with $u \neq 1, \lambda \neq 1, u \neq \lambda$ and $0<x<1$.

For parameters $\lambda, u \in \mathbb{C}, u \neq \lambda$ and $a, b, c \in \mathbb{R}^{+}$whit $a \neq b$, of generalized Apostol-type Frobenius-Euler polynomials are defined by means of the following generating functions (see [18, p. 9, Definition 4.1]):

$$
\left(\frac{a^{z}-u}{\lambda b^{z}-u}\right) c^{x z}=\sum_{n=0}^{\infty} \mathfrak{H}_{n}(x ; a, b, c ; u ; \lambda) \frac{z^{n}}{n !}, \quad|z|<\left|\frac{\log \left(\frac{\lambda}{u}\right)}{\ln b}\right|,
$$

if $x=0$ in (9) then we get $\mathfrak{H}_{n}(a, b, c ; u ; \lambda)$, which denotes the generalized Apostol-type Frobenius-Euler numbers (see $[18$, p. 9]).

For $n=0$ and $a, b \in \mathbb{R}^{+}$, whit $a \neq b, u, \lambda \in \mathbb{C}$ with $u \neq \lambda$ it is then true that $\mathfrak{H}_{n}(a, b ; u ; \lambda)=$ $\frac{1-u}{\lambda-u}$. For $n>0$ we have (see [18, p. 10, Theorem 4.2])

$$
\lambda(\ln b+\mathfrak{H}(a, b ; u ; \lambda))^{n}-u \mathfrak{H}_{n}(a, b ; u ; \lambda)=(\ln a)^{n} .
$$


As an example, the generalized Apostol-type Frobenius-Euler numbers and polynomials are (with the help of MAPLE) as follows.

The generalized Apostol-type Frobenius-Euler numbers:

$$
\begin{aligned}
\mathfrak{H}_{0}(a, b ; u ; \lambda) & =\frac{1-u}{\lambda-u}, \\
\mathfrak{H}_{1}(a, b ; u ; \lambda) & =\frac{\ln a-\lambda \ln b}{(\lambda-u)}, \\
\mathfrak{H}_{2}(a, b ; u ; \lambda) & =\frac{(\ln a)^{2}-\lambda(\ln b)^{2}}{(\lambda-u)}-\lambda \ln b \frac{\ln a-\lambda \ln b}{(\lambda-u)^{2}} .
\end{aligned}
$$

The generalized Apostol-type Frobenius-Euler polynomials:

$$
\begin{aligned}
& \mathfrak{H}_{0}(x ; a, b, c ; u ; \lambda)=\frac{1-u}{\lambda-u}, \\
& \mathfrak{H}_{1}(x ; a, b, c ; u ; \lambda)=x \frac{\ln c(1-u)}{\lambda-u}+\frac{\ln a-\lambda \ln b}{(\lambda-u)}, \\
& \mathfrak{H}_{2}(x ; a, b, c ; u ; \lambda) \\
& \quad=x^{2} \frac{(\ln c)^{2}(1-u)}{\lambda-u}-2 x \ln c \frac{\ln a-\lambda \ln b}{(\lambda-u)}+\frac{(\ln a)^{2}-\lambda(\ln b)^{2}}{(\lambda-u)}-2 \lambda \ln b \frac{\ln a-\lambda \ln b}{(\lambda-u)^{2}} .
\end{aligned}
$$

These polynomials are commonly said to be of Euler type, and they have been studied by various authors in different applications of practical importance (see $[1,12,21])$.

On the other hand, the Hurwitz-Lerch zeta function $\Phi(z, s, a)$ is defined as (see [15, p. 296, Eq. (4.1)])

$$
\Phi(z, s, a)=\sum_{n=0}^{\infty} \frac{z^{n}}{(n+a)^{s}}, \quad a \in \mathbb{C} \backslash \mathbb{Z}_{0}^{-} ; s \in \mathbb{C} \text { when }|z|<1
$$

and $\Re e(s)>1$ for every $|z|=1$.

For $z=1$ in (10) we have the Hurwitz zeta functions

$$
\zeta(s, a)=\Phi(1, s, a)=\sum_{n=0}^{\infty} \frac{1}{(n+a)^{s}} .
$$

Recently, there was defined a new family of Lerch-type zeta function, interpolating a certain class of higher-order Apostol-type numbers and Apostol-type polynomials (cf. [20]). We will use (10) and (11) in Theorems 5.1 and 5.2.

\section{Fourier expansion of generalized Apostol-type Frobenius-Euler polynomials} $\mathfrak{H}_{\mathfrak{n}}(x, a, b, c ; u ; \lambda)$

In this section, we get the Fourier expansions for the generalized Apostol-type FrobeniusEuler polynomials.

Theorem 3.1 Let $u, \lambda \in \mathbb{C} \backslash\{0 ; 1\} u \neq \lambda$ and $a, b, c \in \mathbb{R}^{+}, 1 \leq a \leq 1.1, b>1,1<c \leq e, 0<x<$ 0.9 , we have

$$
\mathfrak{H}_{\mathfrak{n}}(x, a, b, c ; u ; \lambda)=n !(\ln b)^{n}\left(\frac{u}{\lambda}\right)^{x \frac{\ln c}{\ln b}}\left[\frac{u-\left(\frac{u}{\lambda}\right)^{\frac{\ln a}{\ln b}}}{u}\right] \sum_{k \in \mathbb{Z}} \frac{e^{\frac{2 \pi k x i \ln c}{\ln b}}}{\left[2 \pi k i-\log \left(\frac{\lambda}{u}\right)\right]^{n+1}} .
$$


Proof First we consider $f_{n}(z)=\frac{1}{z^{n+1}} \frac{a^{z}-u}{\lambda b^{z}-u} c^{x z}$ and the following integral:

$$
\int_{C} f_{n}(z) d z
$$

over the circle $C=\left\{z:|z| \leq(2 N+\varepsilon) \pi\right.$ and $\left.\varepsilon \in \mathbb{R},\left(\varepsilon \pi i \pm \log \left(\frac{\lambda}{u}\right) \neq 0(\bmod 2 \pi i)\right)\right\}$.

The poles of the function $f_{n}(z)$ are given by

$$
z_{k}=\frac{2 \pi k i-\log \left(\frac{\lambda}{u}\right)}{\ln b}, \quad k \in \mathbb{Z} .
$$

With $z=0$ a pole of order $n+1$. From the Cauchy residue theorem we have (see [7, p. 112, Theorem 2.2])

$$
\int_{C} f_{n}(z) d z=2 \pi i\left\{\operatorname{Res}\left(f_{n}(z), z=0\right)+\sum_{k \in \mathbb{Z}} \operatorname{Res}\left(f_{n}(z), z=z_{k}\right)\right\} .
$$

We calculate $\operatorname{Res}\left(f_{n}(z), z=0\right)$ and $\operatorname{Res}\left(f_{n}(z), z=z_{k}\right)$ as follows (see [7, p. 113, Proposition 2.4]):

$$
\begin{aligned}
\operatorname{Res}\left(f_{n}(z), z=0\right) & =\lim _{z \rightarrow 0} \frac{1}{n !} \frac{d^{n}}{d z^{n}}\left[(z-0)^{n+1} \frac{1}{z^{n+1}} \sum_{m=0}^{\infty} \mathfrak{H}_{\mathfrak{m}}(x, a, b, c ; u ; \lambda) \frac{z^{m}}{m !}\right] \\
& =\lim _{z \rightarrow 0} \frac{1}{n !} \sum_{m=n}^{\infty} \mathfrak{H}_{\mathfrak{m}}(x, a, b, c ; u ; \lambda) \frac{z^{m-n}}{(m-n) !} \\
& =\frac{\mathfrak{H}_{\mathfrak{n}}(x, a, b, c ; u ; \lambda)}{n !} .
\end{aligned}
$$

Also

$$
\begin{aligned}
\operatorname{Res}\left(f_{n}(z), z=z_{k}\right) & =\lim _{z \rightarrow z_{k}}\left(z-z_{k}\right)(z)^{-(n+1)} \frac{a^{z}-u}{\lambda b^{z}-u} c^{x z} \\
& =\frac{1}{z_{k}^{n+1}}\left(a^{z k}-u\right) c^{x z_{k}} \lim _{z \rightarrow z_{k}} \frac{z-z_{k}}{\lambda b^{z}-u} \\
& =\frac{1}{\left[\frac{2 \pi k x i-\log \left(\frac{\lambda}{u}\right)}{\ln b}\right]^{n+1}}\left(a^{\frac{2 \pi k x i-\log \left(\frac{\lambda}{u}\right)}{\ln b}}-u\right) c^{\frac{2 \pi k x i+\log \left(\frac{u}{\lambda}\right)^{x}}{\ln b}} \frac{1}{\lambda b^{\frac{2 \pi k x i-\log \left(\frac{\lambda}{u}\right)}{\ln b}} \ln b} .
\end{aligned}
$$

So, in (14) we have

$$
\begin{aligned}
\int_{C} f_{n}(z) d z= & 2 \pi i\left\{\frac{\mathfrak{H}_{\mathfrak{n}}(x, a, b, c ; u ; \lambda)}{n !}\right. \\
& \left.+\sum_{k \in \mathbb{Z}} \frac{\left(a^{\frac{2 \pi k x i-\log \left(\frac{\lambda}{u}\right)}{\ln b}}-u\right) c^{\frac{2 \pi k x i+\log \left(\frac{u}{\lambda}\right)^{x}}{\ln b}}}{\left[\frac{2 \pi k x i-\log \left(\frac{\lambda}{u}\right)}{\ln b}\right]^{n+1}} \frac{1}{\lambda b^{\frac{2 \pi k x i-\log \left(\frac{\lambda}{u}\right)}{\ln b}} \ln b}\right\} .
\end{aligned}
$$

Taking $N \rightarrow \infty$ it becomes $\int_{C} f_{n}(z) d z=0$. So we have

$$
\mathfrak{H}_{\mathfrak{n}}(x, a, b, c ; u ; \lambda)=-n ! c^{\log \left(\frac{u}{\lambda}\right)^{x}} \sum_{k \in \mathbb{Z}} \frac{\left[a^{\frac{2 \pi k i-\log \left(\frac{\lambda}{u}\right)}{\ln b}}-u\right] c^{\frac{2 \pi k x i}{\ln b}}}{\left[\frac{2 \pi k i-\log \left(\frac{\lambda}{u}\right)}{\ln b}\right]^{n+1} \lambda b^{\frac{2 \pi k i-\log \left(\frac{\lambda}{u}\right)}{\ln b}} \ln b} .
$$

In (15), as $a, b, c$, are expressed in terms of exponential, we complete the proof. 
Corollary 3.1 Let $u, \lambda \in \mathbb{C}$ with $u \neq 1, \lambda \neq 1, u \neq \lambda ; 0<x<1$ and $a=1, b=c=e$, we have

$$
\mathfrak{H}_{\mathfrak{n}}(x, 1, e, e ; u ; \lambda)=\mathfrak{H}_{\mathfrak{n}}(x ; u ; \lambda)=\frac{(u-1)}{u} \frac{u^{x}}{\lambda^{x}} n ! \sum_{k \in \mathbb{Z}} \frac{e^{2 \pi i k x}}{\left[2 \pi i k-\log \left(\frac{\lambda}{u}\right)\right]^{n+1}} .
$$

This is the Fourier expansion for Apostol-type Frobenius-Euler polynomials (see [2, p. 5, Theorem 1]).

Corollary 3.2 Let $u, \lambda \in \mathbb{C}$ with $u \neq 1, \lambda=1, u \neq \lambda ; 0<x<1$ and $a=1, b=c=e$, we have

$$
\mathfrak{H}_{\mathfrak{n}}(x, 1, e, e ; u ; 1)=\mathfrak{H}_{\mathfrak{n}}(x, 1, u)=\mathfrak{H}_{\mathfrak{n}}(x, u)=\frac{(u-1)}{u} u^{x} n ! \sum_{k \in \mathbb{Z}} \frac{e^{2 \pi i k x}}{[2 \pi i k+\log (u)]^{n+1}} .
$$

This is the Fourier expansion for Frobenius-Euler polynomials obtained in (see $[2, p .8$, Corollary 4]).

Corollary 3.3 Let $u, \lambda \in \mathbb{C}$ with $u \neq 1, u \neq \lambda ; 0<x<1 ; a=1, b=c=e ; u=-1$, we have

$$
\mathfrak{H}_{\mathfrak{n}}(x, 1, e, e ;-1 ; \lambda)=H_{n}(x, \lambda,-1)=\mathfrak{E}_{\mathfrak{n}}(x ; \lambda)=2 n ! \sum_{k \in \mathbb{Z}} \frac{e^{(2 k-1) \pi i k x}}{[(2 k-1) \pi i-\log (\lambda)]^{n+1}} .
$$

This is the Fourier expansion for Apostol-Euler polynomials (see [14, p. 2196, Eq. (2.8)]).

\section{Integral representation of the generalized Apostol-type Frobenius-Euler polynomials}

In this section, we will show the integral representation of generalized Apostol-type Frobenius-Euler polynomials.

Theorem 4.1 For $n \in \mathbb{N}$ and $0<x \leq 0,9,|\xi|<\frac{1}{2}, \xi \in \mathbb{R}, a, b, c \in \mathbb{R}^{+}, 1 \leq a \leq 1,1 b>1$ and $1<c \leq e$,

$$
\begin{aligned}
\mathfrak{H}_{n}\left(x ; a, b, c ; u ;-u e^{2 \pi i \xi}\right)= & \Theta\left[\int_{0}^{\infty} \frac{D(n ; x, v)\left(e^{2 \pi(v-i x \ln c} \ln \right)}{\left.\ln e^{2 \xi \pi v}+e^{-2 \xi \pi v}\right)} v^{n} d v\right] \\
& +\Theta\left[\int_{0}^{\infty} \frac{i B(n ; x, v)\left(e^{2 \pi\left(v-i x \frac{\ln c}{\ln b}\right)} e^{2 \xi \pi v}-e^{-2 \xi \pi v}\right)}{\cosh 2 \pi v-\cos 2 \pi x \frac{\ln c}{\ln b}} v^{n} d v\right],
\end{aligned}
$$

where

$$
\begin{aligned}
& \Theta=\frac{1}{2}(\ln b)^{n}\left[\frac{u-e^{-2 \pi i x \xi \frac{\ln a}{\ln b}}(-1)^{\frac{\ln a}{\ln b}}}{u}\right] e^{-\left(2 \xi \pi i x \frac{\ln c}{\ln b}\right)}, \\
& D(n ; x, v)=\left[e^{\pi v} \cos \left(\pi x \frac{\ln c}{\ln b}-\frac{(n+1) \pi}{2}\right)+e^{-\pi v} \cos \left(\pi x \frac{\ln c}{\ln b}+\frac{(n+1) \pi}{2}\right)\right], \\
& B(n ; x, v)=\left[e^{\pi v} \sin \left(\pi x \frac{\ln c}{\ln b}-\frac{(n+1) \pi}{2}\right)-e^{-\pi v} \sin \left(\pi x \frac{\ln c}{\ln b}+\frac{(n+1) \pi}{2}\right)\right] .
\end{aligned}
$$

Proof From (12) and taking $\lambda=-u e^{2 \pi i \xi}, k \longmapsto-k$ we have

$$
\mathfrak{H}_{\mathfrak{n}}\left(x, a, b, c ; u ;-u e^{2 \pi i \xi}\right)
$$




$$
\begin{aligned}
& =(\ln b)^{n}\left[\frac{u-e^{-2 \pi i x \xi \frac{\ln a}{\ln b}}(-1)^{\frac{\ln a}{\ln b}}}{u}\right] e^{-\pi i x \frac{\ln c}{\ln b}} e^{-2 \pi i \xi x \frac{\ln c}{\ln b}} \frac{n !}{(-\pi i)^{n+1}} \\
& \quad \times \sum_{k \in \mathbb{Z}} \frac{e^{-2 \pi i k x \frac{\ln c}{\ln b}}}{[2 k+2 \xi+1]^{n+1}},
\end{aligned}
$$

using (5) and

$$
\left(\frac{-1}{i}\right)^{n+1}=e^{\frac{(n+1) \pi i}{2}} ; \quad(-1)^{(n+1)}=e^{-(n+1) \pi i},
$$

we have

$$
\begin{aligned}
\mathfrak{H}_{\mathfrak{n}}\left(x, a, b, c ; u ;-u e^{2 \pi i \xi}\right) & \\
= & {\left[\frac{u-e^{-2 \pi i x \xi \frac{\ln a}{\ln b}}(-1)^{\frac{\ln a}{\ln b}}}{u}\right] \frac{(\ln b)^{n}}{(-\pi i)^{n+1}} \sum_{k=0}^{\infty} e^{-\left(2 k \frac{\ln c}{\ln b}+2 \xi \frac{\ln c}{\ln b}+\frac{\ln c}{\ln b}\right) \pi i x} \int_{0}^{\infty} t^{n} e^{-(2 k+2 \xi+1) t} d t } \\
& +\left[\frac{u-e^{-2 \pi i x \xi \frac{\ln a}{\ln b}}(-1)^{\frac{\ln a}{\ln b}}}{u}\right] \frac{(\ln b)^{n}}{(-\pi i)^{n+1}}(-1)^{n+1} \\
& \times \sum_{k=0}^{\infty} e^{\left(2 k \frac{\ln c}{\ln b}-2 \xi \frac{\ln c}{\ln b}-\frac{\ln c}{\ln b}\right) \pi i x} \int_{0}^{\infty} t^{n} e^{-(2 k-2 \xi-1) t} d t .
\end{aligned}
$$

Thus,

$$
\begin{aligned}
& \mathfrak{H}_{\mathfrak{n}}\left(x, a, b, c ; u ;-u e^{2 \pi i \xi}\right) \\
& =\left[\frac{u-e^{-2 \pi i x \xi \frac{\ln a}{\ln b}}(-1)^{\frac{\ln a}{\ln b}}}{u}\right] \frac{(\ln b)^{n} e^{-2 \xi \pi i x \frac{\ln c}{\ln b}}}{(-\pi i)^{n+1}} \int_{0}^{\infty} \frac{e^{-\pi i x \frac{\ln c}{\ln b}}}{e^{2 t}-e^{-2 \pi i x \frac{\ln c}{\ln b}}} e^{(1-2 \xi) t} t^{n} d t \\
& +\left[\frac{u-e^{-2 \pi i x \xi \frac{\ln a}{\ln b}}(-1)^{\frac{\ln a}{\ln b}}}{u}\right] \frac{(\ln b)^{n} e^{-2 \xi \pi i x \frac{\ln c}{\ln b}}}{(-\pi i)^{n+1}}(-1)^{n+1} \int_{0}^{\infty} \frac{e^{-\pi i x \frac{\ln c}{\ln b}}}{e^{2 t}-e^{2 \pi i x \frac{\ln c}{\ln b}}} e^{(3+2 \xi) t} t^{n} d t,
\end{aligned}
$$

then

$$
\begin{aligned}
\mathfrak{H}_{\mathfrak{n}}\left(x ; a, b, c ; u ;-u e^{2 \pi i \xi}\right) & \\
= & \frac{\Theta}{(\pi)^{n+1}}\left\{\int_{0}^{\infty} e^{\frac{(n+1) \pi i}{2}} \mho(t ; x, ; b, c) e^{\pi i x \frac{\ln c}{\ln b}} e^{-(2 \xi-1) t} t^{n} d t\right. \\
& +\int_{0}^{\infty} e^{\frac{-(n+1) \pi i}{2}} \frac{\left(e^{2 \pi i x \frac{\ln c}{\ln b}}-e^{-2 t}\right)}{\cosh (2 t)-\cos \left(2 \pi x \frac{\ln c}{\ln b}\right)} e^{\left.-3 \pi i x \frac{\ln c}{\ln b} e^{(2 \xi+3) t} t^{n} d t\right\}}
\end{aligned}
$$

where

$$
\mho(t ; x, ; b, c)=\frac{\left(e^{-2 \pi i x \frac{\ln c}{\ln b}}-e^{-2 t}\right)}{\cosh (2 t)-\cos \left(2 \pi x \frac{\ln c}{\ln b}\right)}
$$

and

$$
\Theta=\frac{1}{2}(\ln b)^{n}\left[\frac{u-e^{-2 \pi i x \xi \frac{\ln a}{\ln b}(-1)^{\frac{\ln a}{\ln b}}}}{u}\right] e^{-\left(2 \xi \pi i x \frac{\ln c}{\ln b}\right)} .
$$


With $i^{n+1}=e^{\frac{(n+1) \pi i}{2}},(-1)^{n+1}=e^{-(n+1) \pi i}$, and making the substitution $t=\pi v$ and simplifying, we complete the result.

Corollary 4.1 For $n \in \mathbb{N}$ and $0 \leq x \leq 1,|\xi|<\frac{1}{2}, \xi \in \mathbb{R}, a=1, b=c=e$, we obtain the integral representation for the Apostol-type Frobenius-Euler polynomials:

$$
\begin{aligned}
\mathfrak{H}_{n}\left(x ; 1, e, e ; u ;-u e^{2 \pi i \xi}\right)= & \frac{\frac{u-1}{u} e^{-2 \xi \pi i x}}{2}\left\{\int_{0}^{\infty} \frac{D(n ; x, v)\left(e^{2 \pi(v-i x)} e^{2 \xi \pi u}+e^{-2 \xi \pi v}\right)}{\cosh 2 \pi v-\cos 2 \pi x} v^{n} d v\right\} \\
& +\frac{\frac{u-1}{u} e^{-2 \xi \pi i x}}{2}\left\{\int_{0}^{\infty} \frac{\left.i B(n ; x, v) e^{2 \pi(v-i x)} e^{2 \xi \pi v}-e^{-2 \xi \pi v}\right)}{\cosh 2 \pi v-\cos 2 \pi x} v^{n} d v\right\},
\end{aligned}
$$

where

$$
\begin{aligned}
& D(n ; x, v)=\left[e^{\pi v} \cos \left(\pi x-\frac{(n+1) \pi}{2}\right)-e^{-\pi v} \cos \left(\pi x+\frac{(n+1) \pi}{2}\right)\right], \\
& B(n ; x, v)=\left[e^{\pi v} \sin \left(\pi x-\frac{(n+1) \pi}{2}\right)-e^{-\pi v} \sin \left(\pi x+\frac{(n+1) \pi}{2}\right)\right] .
\end{aligned}
$$

Corollary 4.2 For $n \in \mathbb{N}$ and $0 \leq x \leq 1,|\xi|<\frac{1}{2}, \xi \in \mathbb{R}, a=1, b=c=e u=-1$, we have

$$
\begin{aligned}
\mathfrak{H}_{n}\left(x ; 1, e, e ;-1 ; e^{2 \pi i \xi}\right)= & e^{-2 \xi \pi i x}\left\{\int_{0}^{\infty} \frac{D(n ; x, v)\left(e^{2 \pi(v-i x)} e^{2 \xi \pi v}+e^{-2 \xi \pi v}\right)}{\cosh 2 \pi v-\cos 2 \pi x} v^{n} d v\right\} \\
& +e^{-2 \xi \pi i x}\left\{\int_{0}^{\infty} \frac{\left.i B(n ; x, v) e^{2 \pi(v-i x)} e^{2 \xi \pi v}-e^{-2 \xi \pi v}\right)}{\cosh 2 \pi v-\cos 2 \pi x} v^{n} d v\right\},
\end{aligned}
$$

where

$$
\begin{aligned}
& D(n ; x, v)=\left[e^{\pi v} \cos \left(\pi x-\frac{(n+1) \pi}{2}\right)-e^{-\pi v} \cos \left(\pi x+\frac{(n+1) \pi}{2}\right)\right], \\
& B(n ; x, v)=\left[e^{\pi v} \sin \left(\pi x-\frac{(n+1) \pi}{2}\right)-e^{-\pi v} \sin \left(\pi x+\frac{(n+1) \pi}{2}\right)\right] .
\end{aligned}
$$

The result obtained in Corollary 4.2 is a new integral representation for the ApostolEuler polynomials $\mathfrak{H}_{n}\left(x ; 1, e, e ;-1 ; e^{2 \pi i \xi}\right)=\mathfrak{E}_{n}\left(x ; e^{2 \pi i \xi}\right)$.

Next, we obtain the integral representation of Apostol-type Frobenius-Genocchi polynomials.

Theorem 4.2 For $n \in \mathbb{N}, 0 \leq x \leq 1,|\xi|<1 / 2, \xi \in \mathbb{R}$, we have

$$
\begin{aligned}
\mathfrak{G}_{n}^{F}\left(x ; u ;-u e^{2 \pi i \xi}\right) & \\
= & {\left[\frac{u-1}{u}\right] \frac{n}{2} e^{-2 \xi \pi i x}\left\{\int_{0}^{\infty} \frac{D_{1}(n ; x, v)\left(e^{2 \pi(v-i x)} e^{2 \xi \pi v}+e^{-2 \xi \pi v}\right)}{\cosh 2 \pi v-\cos 2 \pi x} v^{n-1} d v\right\} } \\
& +\left[\frac{u-1}{u}\right] \frac{n}{2} e^{-2 \xi \pi i x}\left\{\int_{0}^{\infty} \frac{\left.i B_{1}(n ; x, v) e^{2 \pi(v-i x)} e^{2 \xi \pi v}-e^{-2 \xi \pi v}\right)}{\cosh 2 \pi v-\cos 2 \pi x} v^{n-1} d v\right\},
\end{aligned}
$$

where

$$
D_{1}(n ; x, v)=\left[e^{\pi v} \cos \left(\pi x-\frac{n \pi}{2}\right)-e^{-\pi v} \cos \left(\pi x+\frac{n \pi}{2}\right)\right],
$$


Urieles et al. Advances in Difference Equations

(2020) 2020:534

Page 9 of 14

$$
B_{1}(n ; x, v)=\left[e^{\pi v} \sin \left(\pi x-\frac{n \pi}{2}\right)-e^{-\pi v} \sin \left(\pi x+\frac{n \pi}{2}\right)\right] .
$$

Proof Considering (8), $\lambda=-u e^{2 \pi i \xi}, k \longmapsto-k$, then

$$
\mathfrak{G}_{\mathfrak{n}}{ }^{F}\left(x ; u ;-u e^{2 \pi i \xi}\right)=\left[\frac{u-1}{u}\right] e^{-\pi i x} e^{-2 \pi i \xi x} \frac{n !}{(-\pi i)^{n}} \sum_{k \in \mathbb{Z}} \frac{e^{-2 \pi i k x}}{[2 k+2 \xi+1]^{n}} .
$$

Using (5), and noting that $\frac{-1}{i}=e^{n \pi i / 2},(-1)^{n}=e^{-n \pi i}$, then we have

$$
\begin{aligned}
\mathfrak{G}_{\mathfrak{n}}{ }^{F}\left(x ; u ;-u e^{2 \pi i \xi}\right)= & {\left[\frac{u-1}{u}\right] \frac{n}{(-\pi i)^{n}}\left\{\sum_{k=0}^{\infty} e^{-(2 k+2 \xi+1) \pi i x} \int_{0}^{\infty} t^{n-1} e^{-(2 k+2 \xi+1) t} d t\right.} \\
& \left.+(-1)^{n} \sum_{k=0}^{\infty} e^{-(-2 k+2 \xi+1) \pi i x} \int_{0}^{\infty} t^{n-1} e^{-(2 k-2 \xi-1) t} d t\right\} \\
= & {\left[\frac{u-1}{u}\right] \frac{n}{(-\pi i)^{n}}\left\{\int_{0}^{\infty} \frac{e^{-(2 \xi+1) \pi i x}}{e^{2 t}-e^{-2 \pi i x}} e^{2 t} e^{-(2 \xi+1) t} t^{n-1} d t\right.} \\
& \left.+(-1)^{n} \int_{0}^{\infty} \frac{e^{(2 \xi+1) \pi i x}}{e^{2 t}-e^{2 \pi i x}} e^{2 t} e^{(1+2 \xi) t} t^{n-1} d t\right\} \\
= & \frac{1}{2}\left[\frac{u-1}{u}\right] \frac{n}{\pi^{n}}\left\{\int_{0}^{\infty} e^{\frac{n \pi i}{2}} \frac{\left(e^{-2 \pi i x}-e^{-2 t}\right)}{\cosh 2 t-\cos 2 \pi x} e^{\pi i x} e^{-(2 \xi-1) t} t^{n-1} d t\right. \\
& \left.+\int_{0}^{\infty} e^{\frac{-n \pi i}{2}} \frac{\left(e^{2 \pi i x}-e^{-2 t}\right)}{\cosh 2 t-\cos 2 \pi x} e^{-3 \pi i x} e^{(2 \xi+3) t} t^{n} d t\right\} .
\end{aligned}
$$

Using $\left(\frac{1}{-i}\right)^{n}=e^{\frac{n \pi i}{2}}$ and $(-1)^{n}=e^{-n \pi i}$, making the substitution $t=\pi v$ and simplifying, we complete the proof.

Corollary 4.3 For $n \in \mathbb{N}, 0 \leq x \leq 1,|\xi|<1 / 2, \xi \in \mathbb{R}$, and $u=-1$ we have

$$
\begin{aligned}
\mathfrak{G}_{n}^{F}\left(x ;-1 ; e^{2 \pi i \xi}\right)= & n e^{-2 \xi \pi i x}\left\{\int_{0}^{\infty} \frac{D_{1}(n ; x, v)\left(e^{2 \pi(v-i x)} e^{2 \xi \pi v}+e^{-2 \xi \pi v}\right)}{\cosh 2 \pi v-\cos 2 \pi x} v^{n-1} d v\right\} \\
& +n e^{-2 \xi \pi i x}\left\{\int_{0}^{\infty} \frac{\left.i B_{1}(n ; x, v) e^{2 \pi(v-i x)} e^{2 \xi \pi v}-e^{-2 \xi \pi v}\right)}{\cosh 2 \pi v-\cos 2 \pi x} v^{n-1} d v\right\},
\end{aligned}
$$

where

$$
\begin{aligned}
& D_{1}(n ; x, v)=\left[e^{\pi v} \cos \left(\pi x-\frac{n \pi}{2}\right)-e^{-\pi v} \cos \left(\pi x+\frac{n \pi}{2}\right)\right], \\
& B_{1}(n ; x, v)=\left[e^{\pi v} \sin \left(\pi x-\frac{n \pi}{2}\right)-e^{-\pi v} \sin \left(\pi x+\frac{n \pi}{2}\right)\right] .
\end{aligned}
$$

The result obtained in Corollary 4.3, is the integral representation for the ApostolGnocchi polynomials.

Theorem 4.3 For $n \in \mathbb{N}, 0 \leq x \leq 1,|\xi|<1 / 2, \xi \in \mathbb{R}$, we have

$$
\mathfrak{H}_{n}\left(x ; e^{2 \pi i \xi}\right)=\left[1-e^{-2 \xi \pi i}\right] \frac{e^{2 \xi \pi i x}}{2}\left\{\int_{0}^{\infty} \frac{D_{2}(n ; x, v)\left(e^{\pi i x} e^{2 \xi \pi v}+e^{-\pi i x} e^{-2 \xi \pi v}\right)}{\cosh 2 \pi v-\cos 2 \pi x} v^{n} d v\right\}
$$




$$
+\left[1-e^{-2 \xi \pi i}\right] \frac{e^{-2 \xi \pi i x}}{2}\left\{\int_{0}^{\infty} \frac{i B_{2}(n ; x, v)\left(e^{\pi i x} e^{2 \xi \pi v}-e^{-\pi i x} e^{-2 \xi \pi v}\right)}{\cosh 2 \pi v-\cos 2 \pi x} v^{n} d v\right\}
$$

where

$$
\begin{aligned}
& D_{2}(n ; x, v)=\left[e^{\pi v} \cos \left(\pi x-\frac{(n+1) \pi}{2}\right)-e^{-\pi v} \cos \left(\pi x+\frac{(n+1) \pi}{2}\right)\right], \\
& B_{2}(n ; x, v)=\left[e^{\pi v} \sin \left(\pi x-\frac{(n+1) \pi}{2}\right)+e^{-\pi v} \sin \left(\pi x+\frac{(n+1) \pi}{2}\right)\right] .
\end{aligned}
$$

Proof Returning to (17), setting $u=e^{2 \pi i \xi}, k \longmapsto-k$ and using the well-known integral formula (5), we complete the proof.

Theorem 4.4 For $n \in \mathbb{N}, 0 \leq x \leq 1,|\xi|<1 / 2, \xi \in \mathbb{R}$, we have

$$
\begin{aligned}
\mathfrak{G}_{n}^{F}\left(x ; e^{2 \pi i \xi}\right) & \\
= & {\left[1-e^{-2 \xi \pi i}\right] \frac{n e^{2 \xi \pi i x}}{2}\left\{\int_{0}^{\infty} \frac{D_{3}(n ; x, v)\left(e^{\pi i x} e^{2 \xi \pi v}+e^{-\pi i x} e^{-2 \xi \pi v}\right)}{\cosh 2 \pi v-\cos 2 \pi x} v^{n-1} d v\right\} } \\
& +\left[1-e^{-2 \xi \pi i}\right] \frac{n e^{-2 \xi \pi i x}}{2}\left\{\int_{0}^{\infty} \frac{i B_{3}(n ; x, v)\left(e^{\pi i x} e^{2 \xi \pi v}-e^{-\pi i x} e^{-2 \xi \pi v}\right)}{\cosh 2 \pi v-\cos 2 \pi x} v^{n-1} d v\right\},
\end{aligned}
$$

where

$$
\begin{aligned}
& D_{3}(n ; x, v)=\left[e^{\pi v} \cos \left(\pi x-\frac{n \pi}{2}\right)-e^{-\pi v} \cos \left(\pi x+\frac{n \pi}{2}\right)\right], \\
& B_{3}(n ; x, v)=\left[e^{\pi v} \sin \left(\pi x-\frac{n \pi}{2}\right)+e^{-\pi v} \sin \left(\pi x+\frac{n \pi}{2}\right)\right] .
\end{aligned}
$$

Proof Returning to (4) and setting $u=e^{2 \pi i \xi}, k \longmapsto-k$ and using the well-known integral formula (5), we complete the proof.

\section{Explicit formulas for the generalized Apostol-type Frobenius-Euler polynomials at rational arguments}

In this section, we show the formula in rational arguments of generalized Apostol-type Frobenius-Euler polynomials, Apostol Frobenius-Euler polynomials, Apostol FrobeniusGenocchi polynomials, Frobenius-Genocchi polynomials, Frobenius-Euler polynomials.

Theorem 5.1 For $n, q \in \mathbb{N}, p \in \mathbb{Z}, u \in \mathbb{C}$, with $\Re e u \neq 1, \xi \in \mathbb{R},|\xi|\langle 1,1 \leq a \leq 1.1, b\rangle$ 1 and $1<c \leq e$, we have the formula for the generalized Apostol-type Frobenius-Euler polynomials at rational arguments given by

$$
\begin{aligned}
\mathfrak{H}_{n}( & \left.\frac{p}{q} ; a, b, c ; u ;-u e^{2 \pi i \xi}\right) \\
= & A_{n}(a, b ; u) \frac{n !}{(2 q \pi)^{n+1}}\left\{\sum_{j=1}^{q} \zeta\left(n+1, \frac{2 j+2 \xi-1}{2 q}\right) e^{\left(\frac{(n+1)}{2}-\frac{(2 j+2 \xi+1) p \frac{\ln c}{\ln b}}{q}\right) \pi i}\right. \\
& \left.+\sum_{j=1}^{q} \zeta\left(n+1, \frac{2 j-2 \xi-3}{2 q}\right) e^{\left(\frac{-(n+1)}{2}-\frac{(2 j-2 \xi-3) p \frac{\ln c}{\ln b}}{q}\right) \pi i}\right\}
\end{aligned}
$$


where

$$
A_{n}(a, b ; u)=(\ln b)^{n}\left[\frac{u-e^{i \pi \ln a} \ln b}{u}\right] .
$$

Proof From Eq. (12) and

$$
i^{n+1}=e^{\frac{(n+1) \pi i}{2}}
$$

we get

$$
\begin{aligned}
& \mathfrak{H}_{n}(x ; a, b, c ; u ; \lambda) \\
& =n !(\ln b)^{n}\left(\frac{u}{\lambda}\right)^{x} \frac{\ln c}{\ln b}\left[\frac{u-\left(\frac{u}{\lambda}\right)^{\frac{\ln a}{\ln b}}}{u}\right] i^{n+1}\left[\sum_{k=0}^{\infty} \frac{e^{\left(\left(\frac{n+1}{2}\right) \pi-2 \pi k x \frac{\ln c}{\ln b}\right) i}}{\left[2 \pi i k+\log \left(\frac{\lambda}{u}\right)\right]^{n+1}}\right] \\
& \quad+n !(\ln b)^{n}\left(\frac{u}{\lambda}\right)^{x \frac{\ln c}{\ln b}}\left[\frac{u-\left(\frac{u}{\lambda}\right)^{\frac{\ln a}{\ln b}}}{u}\right] i^{n+1}\left[\sum_{k=0}^{\infty} \frac{e^{\left(-\left(\frac{n+1}{2}\right) \pi+2 \pi k x\right.} \frac{\ln c) i}{\ln b}}{\left[2 \pi i k-\log \left(\frac{\lambda}{u}\right)\right]^{n+1}}\right] .
\end{aligned}
$$

The result shown below is equivalent to (21):

$$
\begin{aligned}
& \mathfrak{H}_{\mathfrak{n}}(x ; a, b, c ; u ; \lambda) \\
& =n !(\ln b)^{n}\left(\frac{u}{\lambda}\right)^{x} \frac{\ln c}{\ln b}\left[\frac{u-\left(\frac{u}{\lambda}\right)^{\frac{\ln a}{\ln b}}}{u}\right] i^{n+1}\left[\sum_{k=1}^{\infty} \frac{e^{\left(\left(\frac{n+1}{2}\right) \pi-(2 k-2) \frac{\ln c}{\ln b} \pi x\right) i}}{\left[2 \pi i k-2 \pi i+\log \left(\frac{\lambda}{u}\right)\right]^{n+1}}\right] \\
& \quad+n !(\ln b)^{n}\left(\frac{u}{\lambda}\right)^{x}\left[\frac{\ln c}{\ln b}\left[\frac{u-\left(\frac{u}{\lambda}\right)^{\frac{\ln a}{\ln b}}}{u}\right] i^{n+1}\left[\sum_{k=1}^{\infty} \frac{e^{\left(\left(-\frac{n+1}{2}\right) \pi+(2 k-2) \frac{\ln c}{\ln b} \pi x\right) i}}{\left[2 \pi i k-2 \pi i-\log \left(\frac{\lambda}{u}\right)\right]^{n+1}}\right] .\right.
\end{aligned}
$$

Thus, according to Eq. (10) and by the elementary identity

$$
\sum_{k=1}^{\infty} f(k)=\sum_{j=1}^{l} \sum_{k=0}^{\infty} f(l k+j), \quad l \in \mathbb{N}
$$

(see [14, p. 2202, Eq. 4.12]) we find the formula

$$
\begin{aligned}
\mathfrak{H}_{\mathfrak{n}}(x ; a, b, c ; u ; \lambda) & \\
= & (\ln b)^{n}\left(\frac{u}{\lambda}\right)^{x} \frac{\ln c}{\ln b}\left[\frac{u-\left(\frac{u}{\lambda}\right)^{\frac{\ln a}{\ln b}}}{u}\right] \\
& \times \frac{n !}{(2 \pi i l)^{n+1}} i^{n+1}\left\{\sum_{j=1}^{l} \Phi\left(e^{-2 l \pi x \ln c} i, n+1, \frac{2 \pi j i+\log \left(\frac{\lambda}{u}\right)}{2 \pi i l}\right) \tau\right. \\
& \left.+\sum_{j=1}^{l} \Phi\left(e^{2 l \pi x \frac{\ln c}{\ln b} i}, n+1, \frac{2 \pi j i-\log \left(\frac{\lambda}{u}\right)}{2 \pi i l}\right) e^{\left(\frac{(-(n+1) \pi}{2}-2 \pi x \frac{\ln c}{\ln b}+2 j \pi x \times \frac{\ln c}{\ln b}\right) i}\right\},
\end{aligned}
$$

where $\tau=e^{\left(\frac{(n+1) \pi}{2}+2 \pi x \ln c-2 j \pi x \frac{\ln c}{\ln b}\right) i}$. Setting $\lambda=-u e^{2 \pi i \xi}, x=\frac{p}{q}, l=q$ in (24), the proof of Theorem 5.1 is completed. 
Corollary 5.1 For $n, q \in \mathbb{N}, p \in \mathbb{Z}, u \in \mathbb{C}$, with $\Re e u \neq 1, \xi \in \mathbb{R},|\xi|<1$, and $a=1, b=c=$ $e$ we have the following formula at rational arguments for the Apostol Frobenius-Euler polynomials:

$$
\begin{aligned}
\mathfrak{H}_{n}( & \left.\frac{p}{q} ; 1, e, e ; u ;-u e^{2 \pi i \xi}\right) \\
= & {\left[\frac{u-1}{u}\right] \frac{n !}{(2 q \pi)^{n+1}}\left\{\sum_{j=1}^{q} \zeta\left(n+1, \frac{2 j+2 \xi-1}{2 q}\right) e^{\left(\frac{(n+1)}{2}-\frac{(2 j+2 \xi+1) p}{q}\right) \pi i}\right.} \\
& \left.+\sum_{j=1}^{q} \zeta\left(n+1, \frac{2 j-2 \xi-3}{2 q}\right) e^{\left(\frac{(n+1)}{2}-\frac{(2 j-2 \xi-3) p}{q}\right) \pi i}\right\} .
\end{aligned}
$$

Theorem 5.2 For $n, q \in \mathbb{N}, p \in \mathbb{Z}, u \in \mathbb{C}$, with $\Re e u \neq 1, \xi \in \mathbb{R},|\xi|<1$, we have the following formula at rational arguments for the Apostol Frobenius-Genocchi polynomials:

$$
\begin{aligned}
\mathfrak{G}_{\mathfrak{n}}{ }^{F}\left(\frac{p}{q} ; u, ;-u e^{2 \pi i \xi}\right)= & {\left[\frac{u-1}{u}\right] \frac{n !}{(2 q \pi)^{n}}\left\{\sum_{j=1}^{q} \zeta\left(n, \frac{2 j-2 \xi-3}{2 q}\right) e^{\left(\frac{(2 j-2 \xi-2) p}{q}-\frac{n}{2}\right) \pi i}\right.} \\
& \left.+\sum_{j=1}^{q} \zeta\left(n, \frac{-2 j+2 \xi-1}{2 q}\right) e^{\left(-\frac{(2 j+2 \xi) p}{q}+\frac{n}{2}\right) \pi i}\right\} .
\end{aligned}
$$

Theorem 5.3 For $n, q \in \mathbb{N}, p \in \mathbb{Z}, \xi \in \mathbb{Z}, u \in \mathbb{C},|\xi|<1$, we have the following formula at rational arguments for the Frobenius-Euler polynomials:

$$
\begin{aligned}
\mathfrak{H}_{\mathfrak{n}}\left(\frac{p}{q} ; e^{2 \pi i \xi}\right)= & {\left[1-e^{2 \pi i \xi}\right] \frac{n !}{(2 q \pi)^{n+1}}\left\{\sum_{j=1}^{q} \zeta\left(n+1, \frac{j+\xi-1}{q}\right) e^{\left(\frac{(2 j+2 \xi-1) p}{q}-\frac{(n+1)}{2}\right) \pi i}\right.} \\
& \left.+\sum_{j=1}^{q} \zeta\left(n+1, \frac{j-\xi-1}{2 q}\right) e^{\left(-\frac{(2 j-2 \xi-1) p}{q}+\frac{(n+1)}{2}\right) \pi i}\right\} .
\end{aligned}
$$

Theorem 5.4 For $n, q \in \mathbb{N}, p \in \mathbb{Z}, u \in \mathbb{C}$, with $\Re e u \neq 1, \xi \in \mathbb{R},|\xi|<1$, the followingformula at rational arguments of Frobenius-Genocchi polynomials:

$$
\begin{aligned}
\mathfrak{G}_{\mathfrak{n}}{ }^{F}\left(\frac{p}{q} ; e^{2 \pi i \xi}\right)= & {\left[1-e^{2 \pi i \xi}\right] \frac{n !}{(2 q \pi)^{n}}\left\{\sum_{j=1}^{q} \zeta\left(n, \frac{j+\xi-1}{q}\right) e^{\left(\frac{(2 j+2 \xi-1) p}{q}-\frac{n}{2}\right) \pi i}\right.} \\
& \left.+\sum_{j=1}^{q} \zeta\left(n, \frac{j-\xi-1}{2 q}\right) e^{\left(-\frac{(2 j-2 \xi-1) p}{q}+\frac{n}{2}\right) \pi i}\right\} .
\end{aligned}
$$

For the proof of Theorems 5.2, 5.3 and 5.4 we use (8), (2) and (10), (4) and the identity (23).

\section{Conclusions}

In this article, we showed the Fourier series representation of generalized Apostol-type Frobenius-Euler polynomials by using the proof of the Cauchy residue theorem. The result presented generalizes several Fourier series representations for polynomial families known to date. Also, we proved an integral representation for this and other known polynomial families. Finally, we presented the explicit formula in rational arguments in terms 
of the Zeta Hurwit Lerch and Zeta Hurwit functions for the generalized Apostol-type Frobenius Euler polynomials also said to be of Euler type.

\author{
Acknowledgements \\ The authors thank the Universidad del Atlántico (Colombia) and the Universidad de la Costa C.U.C. (Colombia) for all the \\ support provided.
}

\title{
Funding
}

Partially supported by the Grants: Internal call for strengthening of research groups. Resolution N. 3511 of August 28, 2019 from Universidad del Atlántico.

Availability of data and materials

Not applicable.

\section{Competing interests}

The authors declare there are no competing interests.

\section{Authors' contributions}

The authors declare that the work was realized in collaboration with the same responsibility. All authors read and approved the final manuscript.

\section{Author details}

${ }^{1}$ Programa de Matemáticas, Universidad del Atlántico, Km 7 Vía Pto, Barranquilla, Colombia. ${ }^{2}$ Departamento de Ciencias Naturales y Exactas, Universidad de la Costa, Barranquilla, Colombia. ${ }^{3}$ Departamento de Ciencias Básicas, Universidad Metropolitana, Barranquilla, Colombia.

\section{Publisher's Note}

Springer Nature remains neutral with regard to jurisdictional claims in published maps and institutional affiliations.

Received: 16 April 2020 Accepted: 18 September 2020 Published online: 29 September 2020

\section{References}

1. Alkan, M., Simsek, Y.: Generating function for q-Eulerian polynomials and their decomposition and applications. Fixed Point Theory Appl. 2013(72), 1 (2013). https://doi.org/10.1186/1687-1812-2013-72

2. Araci, S., Acikgoz, M.: Construction of Fourier expansion of Apostol Frobenius-Euler polynomials and its applications. Adv. Differ. Equ. 2018, 67 (2018). https://doi.org/10.1186/s13662-018-1526-x

3. Bayad, A.: Fourier expansion for Apostol Bernoulli, Apostol Euler and Apostol Genocchi polynomials. Math. Comput. 80, 2219-2221 (2011). https://doi.org/10.1090/S0025-5718-2011-02476-2

4. Bayad, A., Kim, T.: Identities for Apostol-type Frobenius-Euler polynomiasl resulting from the study of a nonlinear operator. Russ. J. Math. Phys. 23, 164-171 (2016). https://doi.org/10.1134/S1061920816020023

5. Cangul, I.N., Cevik, A.S., Simsek, Y.: Generalization of q-Apostol-type Eulerian numbers and polynomials, and their interpolation functions. Adv. Stud. Contemp. Math. 25(2), 211-220 (2015)

6. Carlitz, L.: Eulerian numbers and polynomials. Math. Mag. 32, 247-260 (1959). https://doi.org/10.2307/3029225

7. Conway, J.B.: Functions of One Complex Variables. Springer, Berlin (1978)

8. Cristina, B., Roberto, B.: Fourier expansions for higher-order Apostol-Genocchi, Apostol-Bernoulli and Apostol-Euler polynomialsv. Adv. Differ. Equ. 2020, 346 (2020). https://doi.org/10.1186/s13662-020-02802-x

9. Follan, G.: Fourier Analysis and Its Applications (1992)

10. Kim, T.: An identity of the symmetry for the Frobenius-Euler polynomials associated with the fermionic $p$-adic invariant q-integrals on Zp. Rocky Mt. J. Math. 41, 239-247 (2011)

11. Kucukoglu, I., Simsek, Y.: Identities and relations on the $q$-Apostol type Frobenius-Euler numbers and polynomials. J. Korean Math. Soc. 56(1), 265-284 (2019). https://doi.org/10.4134/JKMS.j180185

12. Kucukoglu, I., Simsek, Y., Srivastava, H.M.: A new family of Lerch-type zeta functions interpolating a certain class of higher-order Apostol-type numbers and Apostol-type polynomials. Quaest. Math. 42 465-478 (2019). https://doi.org/10.2989/16073606.2018.1459925

13. Kurt, B., Simsek, Y.: On the generalized Apostol-type Frobenius-Euler polynomials. Adv. Differ. Equ. 2013, 1 (2013). https://doi.org/10.1186/1687-1847-2013-1

14. Luo, Q.: Fourier expansion and integral representations for the Apostol Bernoulli and Apostol Euler polynomials Math. Comput. 78, 2193-2208 (2009)

15. Luo, Q.-M.: Extensions of the Genocchi polynomials and its Fourier expansions and integral representations. Osaka J. Math. 48, 291-309 (2011)

16. Quintana, Y., Ramírez, W., Urieles, A.: Euler matrices and their algebraic properties revisited. Appl. Math. Inf. Sci. 14(4), 583-596 (2020). https://doi.org/10.18576/amis/140407

17. Ramírez, W., Ortega, M., Urieles, A.: New generalized Apostol Frobenius-Euler polynomials and their matrix approach. Kragujev. J. Math. 45(3), 393-407 (2021)

18. Simsek, Y.: Generating functions for generalized Stirling type numbers, array type polynomials, Eulerian type polynomials and their application. Fixed Point Theory Appl. 2013(87), 1 (2013) https://doi.org/10.1186/1687-1812-2013-87

19. Srivastava, H.M., Choi, J.: Zeta and q-Zeta Functions and Associated Series and Integrals. Elsevier, Amsterdam (2012) 
20. Srivastava, H.M., Kurt, B., Simsek, Y.: Some families of Genocchi type polynomials and their interpolation functions. Integral Transforms Spec. Funct. 23(12), 919-938 (2012). https://doi.org/10.1080/10652469.2011.643627

21. Yilmaz, S.: Generating functions for q-Apostol type Frobenius-Euler numbers and polynomials. Axioms 1(3), 395-403 (2012). https://doi.org/10.3390/axioms1030395

Submit your manuscript to a SpringerOpen ${ }^{\odot}$ journal and benefit from:

- Convenient online submission

$\checkmark$ Rigorous peer review

Open access: articles freely available online

- High visibility within the field

- Retaining the copyright to your article

Submit your next manuscript at $\boldsymbol{\Delta}$ springeropen.com 